\title{
Challenges of Iranian Adolescents for Preventing Dental Caries
}

\author{
Arezoo Fallahi ${ }^{1}$; Fazlollah Ghofranipour ${ }^{1, *}$; Fazlollah Ahmadi ${ }^{2}$; Beheshteh Malekafzali ${ }^{3}$; \\ Ebrahim Hajizadeh ${ }^{4}$ \\ ${ }^{1}$ Department of Health Education, Faculty of Medical Sciences, Tarbiat Modares University, Tehran, IR Iran \\ 2 Department of Nursing, Faculty of Medical Sciences, Tarbiat Modares University, Tehran, IR Iran \\ 3 Department of Pediatrics, Faculty of Dentistry, Shahid Beheshti University of Medical Sciences, Tehran, IR Iran \\ ${ }^{4}$ Department of Pediatrics, Faculty of Dentistry, Shahid Beheshti University of Medical Sciences, Tehran
${ }^{2}$ Bostatistics, Faculty of Medical Sciences, Tarbiat Modares University, Tehran, IR Iran \\ ${ }^{*}$ Corresponding Author: Fazlollah Ghofranipour, Department of Health Education, Faculty of Medical Sciences, Tarbiat Modares University, P. O. Box: 14115-331, Tehran, IR Iran. Tel: +98- \\ 2182883669, Fax: +98-2188013030, E-mail: ghofranf@modares.ac.ir
}

Received: September 23, 2013; Revised: December 25, 2013; Accepted: February 12, 2014

\begin{abstract}
Background: Oral health plays a vital role in people's general health and well-being. With regard to the costly treatments of oral diseases, preventive programs need to be designed for dental caries based on children's perspectives.

Objectives: The purpose of this study was to describe and explore challenges for caring dental health based on children's perspectives. Patients and Methods:A qualitative design with content analysis approach was applied to collect and analyze the perspectives of students about factors influencing oral and dental care. Eighteen Iranian students in 8 guidance schools were chosen through the purposive sampling. Semi-structured interviews were held for data gathering. In order to support the validity and rigor of the data, different criteria such as acceptability, confirmability, and transferability were utilized.

Results: During data analysis, four main themes developed: "barriers to dental health," "maintaining dental health," "uncertainty in decision-making" and "supportive factors". "Uncertainty in decision-making" and "barriers to dental health" were the main challenges for preventing dental caries among adolescents.

Conclusions: "Certainty in decision-making" to maintain dental health depends on overcoming the barriers of dental health. Further research is needed to confirm the findings of this study.
\end{abstract}

Keywords:Qualitative Research; Student; Dental Caries; Dental Health

\section{Background}

Oral health plays a vital role in people's general health and well-being and is a fundamental aspect of health (1). Healthy and free of pain mouth enables an individual to eat, speak, and have higher self-esteem and enjoy quality of life (2).

The two most prevalent diseases in the world are dental caries and periodontal diseases. Tooth decay is the most common chronic health problem of adolescents $(3,4)$. Despite an overall decline in the worldwide prevalence of dental caries, some adolescents suffer from this disease (1). The mean value of decay, missing, and filling tooth (DMFT) among 12-year-old children in the world is 1.61. These values in Europe, Africa, America and West Pacific have been reported to be $2.57,1.3,2.76$, and 1.48, respectively (5). According to WHO (2005), the optimal index of DMFT is 1 among 12-year-old children all over the world (6).

Forty percent of the population in Iran, one of the youngest societies in the world, is younger than 20 years old (3). Whereas youth is an important period in the life and development of oral health behaviors, the oral health behavior of children in Iran is still unsatisfactory
(3). The DMFT index among Iranian children (12-year-old) is 1.9 (7). And the percentage of the children who ignore daily brushing and flossing is $70 \%(8)$. Whereas dental cleaning behaviors are one of the most effective tools for prevention of dental caries (9), it has been shown that development of dental caries depends on socioeconomic, cultural, political and environmental factors (10-12), as well as individual's perceptions and behaviors and personality traits (13).

Owing to the costly treatments of dental diseases (14, 15), the prevalence of dental caries in children (16), need for health promotion programs for students with dental caries (5) and better understanding of defective oral health behaviors (17), designing preventive programs and educational contents based on children's perspective suffering from dental caries is necessary (1).

There were no qualitative studies exploring challenges for caring dental health in the Iranian medical literature. In addition, it is to be noted that a quantitative design could not entirely describe factors influencing dental care. Conducting qualitative studies will help improve our understanding of socioeconomic, cul-

Copyright (C) 2014, Iranian Red Crescent Medical Journal; Published by Kowsar. This is an open-access article distributed under the terms of the Creative Commons Attribution-NonCommercial 4.0 International License (http://creativecommons.org/licenses/by-nc/4.0/) which permits copy and redistribute the material just in noncommercial usages, provided the original work is properly cited. 
tural, political and environmental aspects influencing adolescents' oral behavior. Moreover, it will help design educational programs to prevent dental caries and enhance the effectiveness of educational interventions in this regard.

\section{Objectives}

The findings of this study can be used by health professionals to design operational interventions and by dentists to develop appropriate strategies to improve adolescents' dental health.

\section{Patients and Methods}

A qualitative design with content analysis approach was used to gather and analyze the data. This type of research aims to explore complex phenomena. It is also the most useful tool in understanding people' emotions and perceptions, let alone it is the most common approach employed in health research $(18,19)$.

Eighteen guidance schools were selected using simple random sampling from north (4 schools), south (5 schools), west (4 schools) and east ( 5 schools) of Tehran City in Iran. Purposive sampling method with maximum variation in sampling was utilized to choose 18 schoolaged students with the following inclusion criteria:

- Aged between 11 and 15 years

- Having dental caries

- Reporting at least one of the signs of toothache, bleeding gum, pulled and filled teeth, and/or orthodontics

- Willingness to sign the informed consent to participate in this study

All participants were included in the study, and no one excluded because of the fact that all participants suffered from dental caries and reported at least one of the above- mentioned sings. At the beginning of the study, the first researcher expressed the purpose of research for students and invited them to participate in the study. Of the participants, 11 were male. The participant aged between 11 and 15 years old. They were suffering from at least two dental diseases such as tooth decay, bleeding gums, tooth ache, pulled or filled teeth, and orthodontics.

The study's proposal was approved by the research council affiliated with Tarbiat Modares University (No: 52.127819) which corroborated its ethical considerations. Permission to enter the research zones were obtained from the school's principals. All the students were informed about the aim and methods of the study, the confidentiality of their identities, and withdrawing from the study at any time. Those students who agreed to willingly participate in the study signed written informed consent. The interviews were conducted in classrooms and consulting rooms in schools.

Face to face semi-structured interviews, group discussions in Persian, and memo writing were held to collect data by the first researcher who was trained to diagnose and identify tooth decay and gum diseases. Also she received some skills in interview techniques and was a PhD student in health education at the time of the study. The main foci of the interviews were describing the experiences of oral disease (bleeding gums, missing and pulled teeth and orthodontics), and how they deal with this situation.

In addition, the participants' thoughts were followed using probing questions. They were asked to clarify their responses, bring examples of the phenomenon and explain the reason for their answers. The duration of the interviews was between 20 and 45 minutes. The sampling was continued until data saturation was reached. After 17 and 18 interview sessions, saturation was reached. A situation that collected information did not add new variations or codes to the findings $(20,21)$.

A content analysis approach was used to analyze the data. Content analysis is suitable to analyze and interpret a large amount of textual data, identify codes and themes through the systematic classification of the text content and earn an understanding of the "themes" and details in the text (22). The following steps were taken to analyze the data:

- Researchers immersed themselves in the raw data through transcribing interviews and reading them several times to get the sense of the whole.

- The participants' remarks and connotations were coded; all codes were then checked by the authors (Indexing) (23).

- The codes were classified and compared based on their similarities and differences (identifying a thematic framework) (23).

- The description of the coding tree was provided (charting) (23).

- The latent meanings of the text as themes were developed. The charts were used to describe the range of participants' views in each theme and subtheme, and the findings linked back to the literature (mapping and interpretation) (23).

As member checking, the participants reviewed a summary of the interviews and their primary analysis and confirmed that the researchers presented their real words. Also participants provided feedback on the findings (credibility) (24). The research team conduced the analysis independently (3 coders), compared their findings and in cases of disagreement; they held discussions until they reached an agreement. For peer checking, three faculty members (research methodologist, dentist, and educational expert) reviewed the analysis report and confirmed it (conformability) (24). Factors of prolonged engagement, constant comparative analysis, audit trail, and a transparent description of the research steps assured that the findings were consistent and could be repeated (dependability) (24). Variance according to sex and age, socioeconomic status, parent's job, education and residential indicated that the findings were applicable in other contexts (transferability) (24). 
Fallahi A et al.

\section{Results}

This study was finalized with 18 students. Of them, 4 students suffered from toothache and dental caries, 3 students from dental caries and bleeding gum, 6 students from dental caries, pulled and filled teeth, 3 students from dental caries, toothache, and orthodontics and 2 students were suffered from dental caries, bleeding gum, and pulled teeth. Educational level of students' parents were primary school ( 2 mothers, 1 father), diploma $(9,10)$, and bachelor's degree ( 6 mothers, 6 fathers) and above. Demographic characteristics of the participants are presented in Table 1. During data analysis, four main themes developed: "barriers to dental health", "maintaining dental health", "uncertainty in decision-making”, and "supportive factors". These four themes explain challenges and strategies for caring dental health based on adolescents' perspectives. It is noted that 10 subthemes were also developed for the themes (Figure 1). The meaning of each theme/subtheme is explained using participants' direct quotations.

\subsection{Challenges for Preventing Dental Caries}

"Barriers to dental health", "uncertainty in decisionmaking" and "supportive factors" were challenges, while "maintaining dental health" was a strategy for caring dental health among Iranian adolescent.

\subsubsection{Barriers to Dental Health (Category 1)}

Subcategories were "external conditions" and "perceived inability".

In this study, external conditions such as an inappropriate perception of the causes of dental caries, lack of attention, knowledge and skills related to dental health were reasons for not brushing teeth. The students did not have information about the roles of deciduous teeth, onset age of dental care, and the role of nutrition in the development of dental caries. For instance, their knowledge about the importance of deciduous teeth and their importance on speech and permanent teeth was low. Lack of attention to deciduous teeth and not brushing them during childhood could be the reason for not brushing and flossing permanent teeth in adolescence.

"I brush my front teeth that are permanent. My posterior teeth are milk teeth and will fall, so I do not brush them" (A 14-year-old girl).

The participants declared that they did not enjoy brushing their teeth, because it was a boring task. They mentioned that their teeth were healthy and need not to be brushed. A difficult condition for the provision of care was described by the students as irksome activities, being busy, forgetting to do the tasks and a low priority, lack of knowledge and skills related to dental health.

Probably, students were dissatisfied with their own careless behaviors, because tooth decay or a threatening condition to their general health would be developed.
For these students, decision-making and behavioral changes were difficult. If they had confidence to clean their teeth, they would admit the responsibility of their dental health by themselves and overcome barriers.

"I feel lazy and tired. I don't have time and often forgot brushing" (A 12-year-old girl).

"When I floss or brush my teeth, my gum begins bleeding; therefore, I don't floss or brush my teeth" (A 14-yearold boy).

The participants stated that dental caries were associated with perceived inability and uncontrollable characteristics such as inheritance and dental fear. They also had negative viewpoints toward dental care and believed that the causes of dental caries were uncontrollable. This negative viewpoint affected students' self-confidence on oral health behaviors.

"Some people say that oral and dental diseases are hereditary, why I bother myself and try to take care of my teeth" (A 12-year-old girl).

Most adolescents mentioned that dental fear was an important reason to avoid the dentist and regular treatment. Dental fear includes fear of dentist, fear of treatment, and fear of dental instruments. They are learned by personal experiences, friends, family members, relatives, and the mass media. The students said that they were unable to deal with the terrible fear and stress before going to the dentist.

"I am scared of the dentist, his clothing, and his appliance" (A 13-year-old girl).

\subsubsection{Maintaining Dental Health (Category 2)}

This category consisted of the following subcategories: "role of mouth and teeth" and "dental care behaviors". The participants mentioned that their mouth and teeth

Table 1. Demographic Characteristics of Participants in the Study

\begin{tabular}{|c|c|}
\hline Variable & Value \\
\hline Age, $y$, mean $\pm S D$ & $13 \pm 0.78$ \\
\hline \multicolumn{2}{|l|}{ Sex } \\
\hline Female & 7 \\
\hline Male & 11 \\
\hline \multicolumn{2}{|c|}{ Types of Guidance schools } \\
\hline Public & 5 \\
\hline Private & 3 \\
\hline \multicolumn{2}{|c|}{ Socioeconomic Status } \\
\hline Low & 9 \\
\hline Average & 5 \\
\hline High & 4 \\
\hline \multicolumn{2}{|l|}{ Insurance } \\
\hline Yes & 13 \\
\hline No & 5 \\
\hline
\end{tabular}


Fallahi A et al.

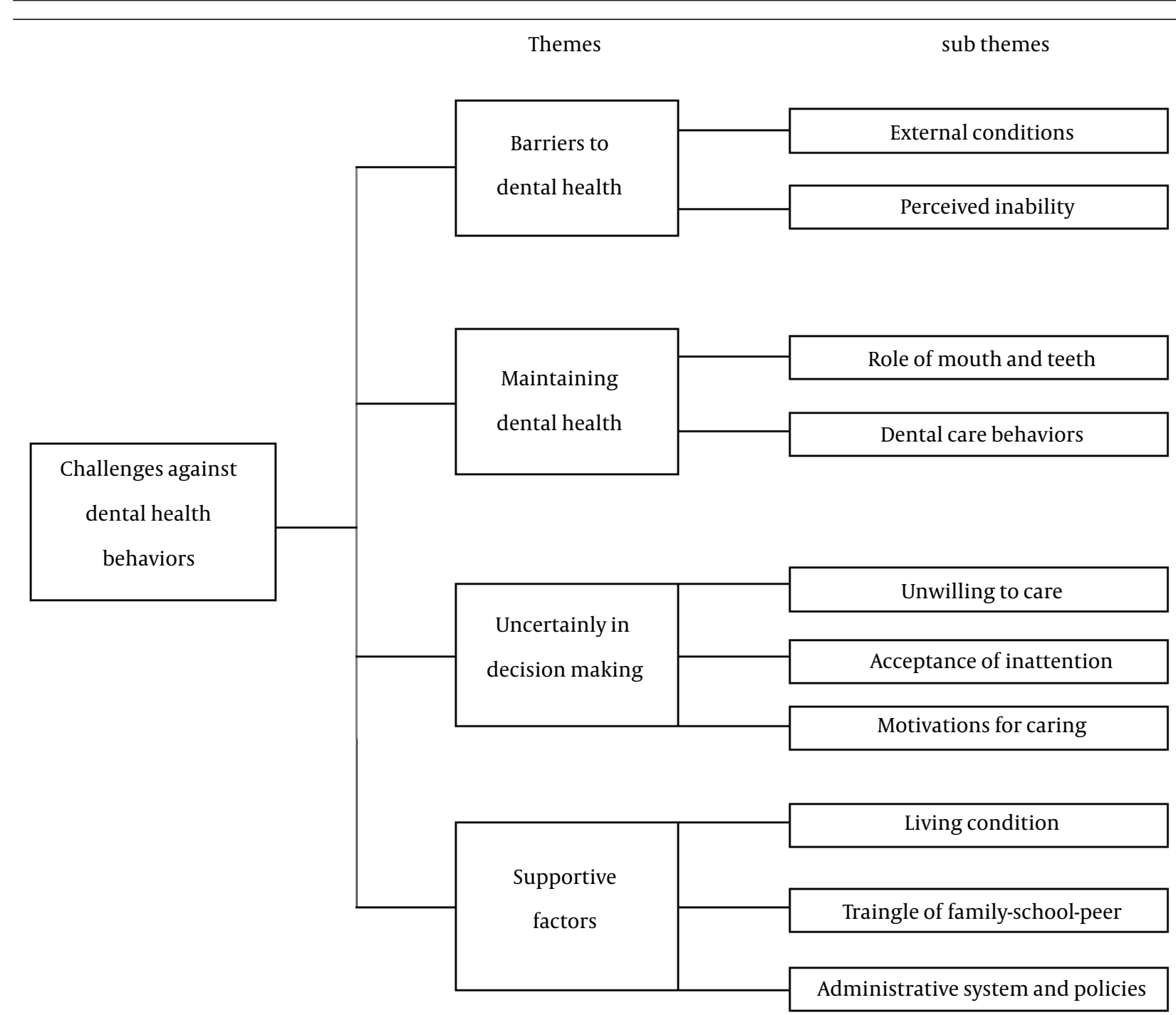

Figure 1. Themes /Subthemes Related to Challenges and Strategy for Caring Dental Health

were an important part of their lives. They stated that healthy mouth affected their confidence, self-image, and self-esteem, eating, speaking, and digestion. Also the adolescents noted that dental healthcare improved their overall health and reduced the risk of serious diseases and even promoted their quality of life. Some students were aware of the role of mouth and teeth on their overall health.

"I look attractive and interesting with my clean and nice teeth. I take care of them" (A13-year-old girl).

"Tooth decay causes bad breath of mouth. I had bad breath of mouth for months. I did not like to speak, because I felt that my friends ignored me and did not like me" (An 11-year-old boy).

The students strived to avoid tooth decay and keep their teeth healthy. The reaction of adolescents towards dental caries and pain was brushing and/or going to the dentist. The students' strategies for maintaining their teeth healthy were brushing, flossing, scaling, avoiding highsugar food, using mouth wash, filling teeth, eating apple, orthodontia, checkup, and endodontic. The main reason for referral to the dentist was tooth decay and dental pain.

"I am brushing my teeth with fluoride toothpaste twice a day for at least two minutes" (A12-year-old girl).

\subsubsection{Uncertainty in Decision-Making (Category 3)}

This category consisted of "reluctant to care", "acceptance of inattention," and "motivations for caring".

"Unwillingness to care" was a barrier in decision-making for brushing and flossing teeth, and a problem in students who are unaware of how to tackle it. Lack of motivation and enthusiasm were two main reasons for ignoring the teeth brushing habit. The students came up with many excuses why they did not or could not brush or floss, and resisted parents' pressure for cleaning their teeth. 
Fallahi A et al.

"My mom says to brush every day, but I do not do it, and I don't pay attention to boring things" (An 11-year-old boy). Acceptance of inattention was a strong element in decision-making for brushing and flossing teeth. The participants stated that there was a relationship between dental caries and inattention to dental health. Discomfort related to tooth decay was an important factor affecting decision-making in dental care and changing unhealthy behaviors.

The participants noted that common social beliefs and religious and social motivators affected their decisionmaking regarding oral heath behaviors and dental care. The students connected oral health behaviors such as brushing and flossing with social and religious beliefs. On the other hand, religious and social motivators and unwillingness to follow dental health behaviors caused uncertainty in students' decision-making regarding cleaning their teeth.

"God has given me teeth, so I have to keep them clean" (an 11-year-old boy).

"All people pay attention to nice teeth, and I try to keep them clean" (a 12-year-old girl).

\subsubsection{Supportive Factors (Category 4)}

This category has three subcategories, including "living condition", "triangle of family-school-peer", and "administrative system and policies". These factors affected students' dental health behaviors that both encouraged and discouraged students to follow healthy behaviors.

The participants stated that any change in the living condition could lead to changes in students' behavioral habits. The participants stated that their family's cultural and socioeconomic status was a critical factor for changing behaviors and referring the dentist.

Poor socioeconomic status of families increases the risk of dental caries and oral diseases.

"Some people use a matchstick or knife to remove food from between their teeth, while they can afford to buy a toothbrush, the culture of oral health among people is low" (a 13-year-old girl).

The triangle of family-school-peer meant that the family, school, and peer could encourage students to clean their teeth and go to the dentist. The interaction between the student and these factors can result in dental cleaning behaviors.

“When I saw my friend who wasn't brushing his teeth, I decided not to clean my teeth, too" (an 11-year-old boy).

"In school, there is no place to brush my teeth" (a 13-yearold boy).

"The educator did not say anything about dental care" (a 13-year-old girl).

“My mom isn't sensitive to my dental care, thus I don't keep my teeth clean" (a 14-year-old boy).

According to the participants, the health care system policies such as insurance, educational system, role of dentist and media were the strongest factors in encouraging people to follow dental care behaviors and follow the treatment of dental caries. In this respect, the provision of public healthcare facilities was deemed important. Also, the media provide learning opportunities through advertisements on the promotion of healthy dental behaviors.

"I am unable to pay for dental care costs. There is a need for public support facilities to cover some costs. The media do not play their role to motivate people to follow healthy behaviors" (a 13-year-old boy).

The participants mentioned that the dentist was responsible to inform them about the importance of brushing and flossing in dental care. However, the dentist would prefer treatment rather than prevention.

"I learned how to floss my teeth for the first time from a dentist" (a 12-year-old girl).

\section{Discussion}

This study was the first qualitative research conducted to explore challenges and strategies for caring dental health among Iranian adolescent. In this study, barriers were the main factors to do oral health behavior. Some adolescents stated that they did not brush and floss their teeth. Low priority for dental care, having no plan and time to follow health behaviors, having dental fear, tiredness, forgetfulness, and lack of knowledge and skill were the reasons for unwillingness to change behaviors or referral to the dentist. Similarly, Pakpour et al. in Iran described that the reasons for ignoring oral health behaviors were tiredness, forgetfulness and having no plan or time to follow health behaviors (1).

The adolescents believed that they could not prevent oral diseases and stated that prevention of tooth decay was not associated with following health behaviors. In a qualitative research in Sweden, adolescents stated that their dental caries was uncontrollable, and hereditary (25). In Iran, Pakpour et al. mentioned that there was a relationship between controllable dental caries and selfefficacy (1). Furthermore, greater perceived control leads to a stronger intention to perform oral heath behaviors that consequently can reduce the risk of dental carries (26). Other main reasons for ignoring dentists were fear of treatment; fear of dentists, individual's unpleasant experiences and other people's unpleasant experiences during referral to the dentist. Negative perceptions of oral health and having no willingness and motivation to accomplish healthy behaviors are the causes of dental caries. These reasons can be causes of uncertainty in decision-making.

There are several strategies to maintain and promote oral health such as increasing knowledge and skills regarding dental health and applying the process of coping with stress. In addition, developing national guidelines would be useful. Schools and healthcare organizations can emphasize on the gingival index and plaque index before adolescents' enrolment in the primary schools and employment.

Some adolescents applied behavioral strategies such as 
dental restoration, root canal treatment, , scaling, brushing, flossing, as well as using mouthwash to prevent the spread of tooth decay, dental pain, and oral diseases. The major problems and the most important reasons for seeking dental care and visiting the dentist were pain due to tooth decay, periodontal diseases, or injury. This finding is consistent with the study of Mashoto et al. in Tanzania that dental pain was a main reason for brushing and dental treatment in adolescents (27).

Dental appearance, nice smile, good speaking, better chewing, enhancement of self-image and self-esteem among Iranian adolescents were other motivators for dental care in the adolescents. Similarly, Trulsson et al. in Sweden reported that teenager's reasons to undergo orthodontic treatment were dental appearance, self-image, and self-esteem (19).

The result of this study indicated that decision-making was affected by factors such as unwillingness to care, acceptance of inattention and motivations for caring. Religious and social motivations affect the adolescents' decision-making regarding oral health. However, these were not strong enough to change individuals' attitudes and behaviors toward dental care. In designing educational programs, educators should attend to these motivational factors. Luzzi and Spencer in Australia reported that existence of religious and social beliefs in the physical environment impacted adolescents' decision-making for oral health (28). Ostberg in his research showed that worries the developed by inattention to teeth was the main factor for practicing oral health behaviors (29). However, he did not mention the role of belief in teeth care. Adolescents should believe that they are responsible for their teeth care.

The results of the present study showed that perceived tooth decay was under the influence of culture, life style, family members, dentist, school, and the mass media. Suggested strategies to increase the confidence in control of dental carries include enhancing adolescents' perception of risk factors in developing dental caries by the family, dentist, and school and changing incorrect social beliefs through designing appropriate educational programs $(28,30,31)$. Some studies showed that most adolescents start oral health behaviors, when they experience oral diseases $(1,29)$.

According to our findings, external factors can also be more effective when adolescents have some previous experiences regarding oral diseases and low perceptions of oral health. The triangle of the family-school-peer, proper education, administrative system and policies, and socioeconomic status decreased unhealthy behaviors and improved healthy dental behavior. Luzzi and Spencer in Australia and Fallahi et al. in Iran reported that the physical environment and family and peer impacted adolescents' decision-making for oral health $(28,32)$. Moreover, Solhi et al. in Iran showed that the mass media, interaction between people, and experiencing some form of the health problem facilitated decision-making on oral health in adolescents (33).
The findings of this research emphasized on the weak effect of education in dental health behaviors in schools. The participants expressed that education of dental health did not sufficiently affect the prevention of tooth decay. In line with our findings, Watt in the UK reported that interventions related to improving oral health were unsuccessful in adolescents and had no effect on the prevention of dental caries (14). When education related to dental health is poorly presented, the student's perception of oral health and confidence to do dental health will decline.

This study showed that certainty in decision-making on maintaining dental health depends on overcoming the barriers of dental health. Understanding adolescents' views and experiences of dental healthy behavior is of vital importance in designing educational models and delivering appropriate educational programs for prevention of tooth decay in adolescents. While generalization is not the aim of qualitative studies, the findings of this study could be transferred to other contexts and cultures with caution and after conducting similar studies. Further research is needed to explore the process of developing dental carries and dental care among people with different cultures and contexts to establish the findings of this study. To identify the strengths and weaknesses of the study, researchers in their countries can design children's oral health program based on the findings of this study and compare the effect of this oral health training program with other training programs.

\section{Acknowledgements}

The authors would like to thank the participants whose assistance resulted in carrying out this research.

\section{Authors' Contributions}

Arezoo Fallahi, Fazlollah Ghofranipour, and Fazlollah Ahmadi: data collection and analysis and manuscript writing; and Beheshteh Malekafzali, and Ebrahim Hajizadeh: data analysis.

\section{Financial Disclosure}

Authors declared no financial interests regarding the materials in the manuscript.

\section{References}

1. Pakpour AH, Hidarnia A, Hajizadeh E, Kumar S, Fridlund B. Why Iranian adolescents do not brush their teeth: a qualitative study. Int J Dent Hyg. 2012;10(2):86-90.

2. Pakpour AH, Hidarnia A, Hajizadeh E, Kumar S, Harrison AP. The status of dental caries and related factors in a sample of Iranian adolescents. Med Oral Patol Oral Cir Bucal. 2011;16(6):e822-7.

3. Morowatisharifabad $M$, Fallahi A, Nadrian $H$, Haerian A Neamatshahrbabaki B. Inter-dental cleaning behavior and its relationship with psychological constructs based on the Transtheoretical model. Prev Dentis J. 2011;9(3):211-20.

4. Pakpour AH, Hidarnia A, Hajizadeh E, Plotnikoff RC. Action and coping planning with regard to dental brushing among Iranian adolescents. Psychol Health Med. 2012;17(2):176-87. 
Fallahi A et al.

5. Hashemian M, Falahi A, Tavakoli G, Zarezadeh Y, Nemat Shahr Babaki B, Rahaei Z. Study of the Impact of Education on InterDental Cleaning behavior based onTrans-Theoretical Model. Oral Prev Den J. 2012;1:37-46.

6. Petersen PE, Bourgeois D, Ogawa H, Estupinan-Day S, Ndiaye C. The global burden of oral diseases and risks to oral health. Bull World Health Organ. 2005;83(9):661-9.

7. Pakshir HR. Oral health in Iran. Int Dent J. 2004;54(6 Suppl 1):36772.

8. Kazemnejad A, Zayeri F, Rokn AR, Kharazifard MJ. Prevalence and risk indicators of periodontal disease among high-school students in Tehran. East Mediterr Health J. 2008;14(1):119-25.

9. Vehkalahti MM, Widstrom E. Teaching received in caries prevention and perceived need for Best Practice Guidelines among recent graduates in Finland. Eur J Dent Educ. 2004;8(1):7-11.

10. Petersen PE. Sociobehavioural risk factors in dental caries international perspectives. Community Dent Oral Epidemiol. 2005;33(4):274-9.

11. Newton JT, Bower EJ. The social determinants of oral health: new approaches to conceptualizing and researching complex causal networks. Community Dent Oral Epidemiol. 2005;33(1):25-34

12. Bastos JL, Peres MA, Peres KG, Araujo CL, Menezes AM. Toothache prevalence and associated factors: a life course study from birth to age 12 yr. Eur J Oral Sci. 2008;116(5):458-66.

13. Atchison KA, Dubin LF. Understanding health behavior and perceptions. Dent Clin North Am. 2003;47(1):21-39.

14. Watt RG. Strategies and approaches in oral disease prevention and health promotion. Bull World Health Organ. 2005;83(9):711-8.

15. Sheiham A, Watt RG. The common risk factor approach: a rational basis for promoting oral health. Community Dent Oral Epidemiol. 2000;28(6):399-406.

16. Bagramian RA, Garcia-Godoy F, Volpe AR. The global increase in dental caries. A pending public health crisis. Am J Dent 2009;22(1):3-8.

17. Kawamura M, Takase N, Sasahara H, Okada M. Teenagers' oral health attitudes and behavior in Japan: comparison by sex and age group.J Oral Sci. 2008;50(2):167-74.

18. Watt RG. Motivational interviewing may be effective in denta setting. Evid Based Dent. 2010;11(1):13.

19. Trulsson U, Strandmark M, Mohlin B, Berggren U. A qualitative study of teenagers' decisions to undergo orthodontic treatment with fixed appliance. J Orthod. 2002;29(3):197-204.

20. Struss A, Corbin J. Basic of qualitative research: Techniques and procedures for developing grounded theory. 3 ed: Sage publication; 2007.

21. Hilton IV, Stephen S, Barker JC, Weintraub JA. Cultural factors and children's oral health care: a qualitative study of carers of young children. Community Dent Oral Epidemiol. 2007;35(6):429-38.

22. Hsieh HF, Shannon SE. Three approaches to qualitative content analysis. Qual Health Res. 2005;15(9):1277-88.

23. Bryman A, Burgess R. Analyzing Qualitative Data. In: Routledge editor; 1994.

24. Guba EG, Lincoln YS. Improving the usefulness of evaluation results through responsive and naturalistic approaches. San Francisco: Jossey-Bass; 1981.

25. Hattne K, Folke S, Twetman S. Attitudes to oral health among adolescents with high caries risk. Acta Odontol Scand. 2007;65(4):206-13.

26. Syrjala AM, Niskanen MC, Knuuttila ML. The theory of reasoned action in describing tooth brushing, dental caries and diabetes adherence among diabetic patients. J Clin Periodontol. 2002;29(5):427-32.

27. Mashoto KO, Astrom AN, David J, Masalu JR. Dental pain, ora impacts and perceived need for dental treatment in Tanzanian school students: a cross-sectional study. Health Qual Life Outcomes. 2009;7:73.

28. Luzzi L, Spencer AJ. Factors influencing the use of public dental services: an application of the Theory of Planned Behaviour. BMC Health Serv Res. 2008;8:93.

29. Ostberg AL, Jarkman K, Lindblad U, Halling A. Adolescents' perceptions of oral health and influencing factors: a qualitative study. Acta Odontol Scand. 2002;60(3):167-73.

30. Saied-Moallemi Z, Virtanen JI, Vehkalahti MM, Tehranchi A, Murtomaa H. School-based intervention to promote preadolescents' gingival health: a community trial. Community Dent Oral Epidemiol. 2009;37(6):518-26.

31. Badri Gargari R, Salek Hadadian N. The role of self-efficacy and factors of Health Belief Model in dental patients' brushing and flossing. Urmia NurMidwifery Fac J. 2011;9(3).

32. Fallahi A, Ghofranipour F, Ahmadi F, Malekafzali B, Hajizadeh E. Adolescent's perspectives on the factors that influence caries development: A qualitative study Scientific. Sch Health Res J. 2013;10(4):65-80.

33. Solhi M, Zadeh DS, Seraj B, Zadeh SF. The application of the health belief model in oral health education. Iran J Public Health. 2010;39(4):114-9. 